KEK-CP-329

\title{
Chiral behavior of light meson form factors in $2+1$ flavor QCD with exact chiral symmetry
}

\author{
JLQCD Collaboration: T. Kaneko ${ }^{\star a, b}$, S. Aoki ${ }^{c, d}$, G. Cossu $^{a}$, X. Feng ${ }^{e}$, H. Fukaya ${ }^{f}$, \\ S. Hashimoto ${ }^{a, b}$, J. Noaki ${ }^{a}$, T.Onogi ${ }^{f}$
}

${ }^{a}$ High Energy Accelerator Research Organization (KEK), Ibaraki 305-0801, Japan

${ }^{b}$ School of High Energy Accelerator Science, SOKENDAI (The Graduate University for Advanced Studies), Ibaraki 305-0801, Japan

c Yukawa Institute for Theoretical Physics, Kyoto University, Kyoto 606-8502, Japan

${ }^{d}$ Center for Computational Sciences, University of Tsukuba, Ibaraki 305-8577, Japan

${ }^{e}$ Physics Department, Columbia University, New York, NY 10027, USA

${ }^{f}$ Department of Physics, Osaka University, Osaka 560-0043, Japan

\begin{abstract}
We present a study of chiral behavior of light meson form factors in QCD with three flavors of overlap quarks. Gauge ensembles are generated at single lattice spacing $0.12 \mathrm{fm}$ with pion masses down to $300 \mathrm{MeV}$. The pion and kaon electromagnetic form factors and the kaon semileptonic form factors are precisely calculated using the all-to-all quark propagator. We discuss their chiral behavior using the next-to-next-to-leading order chiral perturbation theory.
\end{abstract}

The 33rd International Symposium on Lattice Field Theory

14 - 18 July 2015

Kobe International Conference Center, Kobe, Japan*

${ }^{*}$ Speaker.

†E-mail: takashi.kaneko@kek.jp 


\section{Introduction}

The $K \rightarrow \pi l v$ semileptonic decays provide a precise determination of the Cabibbo-KobayashiMaskawa matrix element $\left|V_{u s}\right|$. Lattice QCD plays an important role to evaluate the normalization of the vector and scalar form factors $f_{+}(0)=f_{0}(0)$ in the $K \rightarrow \pi$ matrix element

$$
\left\langle\pi\left(p^{\prime}\right)\left|V_{\mu}\right| K(p)\right\rangle=\left(p+p^{\prime}\right)_{\mu} f_{+}(t)+\frac{M_{K}^{2}-M_{\pi}^{2}}{t} q_{\mu}\left\{f_{0}(t)-f_{+}(t)\right\} \quad\left(t=q^{2}=\left(p-p^{\prime}\right)^{2}\right) .
$$

The required accuracy is high, typically within $1 \%$. The phase space integral for the decay rate is estimated from the form factor shape, namely their $t$ dependence, which has been precisely measured by experiments. Therefore a rigorous comparison of the shape between lattice QCD and experiments can demonstrate the reliability of the precision calculation of $f_{+}(0)$. This article presents a lattice calculation of these form factors and analysis based on next-to-next-to-leading order (NNLO) chiral perturbation theory (ChPT). We also discuss the kaon and pion electromagnetic (EM) form factors defined through

$$
\left\langle P\left(p^{\prime}\right)\left|J_{\mu}\right| P(p)\right\rangle=\left(p+p^{\prime}\right)_{\mu} F_{V}^{P}(t) \quad\left(P=\pi^{+}, K^{+}, K^{0}\right),
$$

which provide helpful information for the ChPT analysis of the semileptonic form factors.

\section{Simulation method}

We simulate $2+1$ flavor QCD using the overlap quark action, which exactly preserves chiral symmetry and enables us to directly compare the lattice data with ChPT. Numerical simulations are remarkably accelerated by modifying the Iwasaki gauge action [1] and by simulating the trivial topological sector $[1,2]$. Note that effects of the fixed global topology can be considered as finite volume effects suppressed by the inverse lattice volume [2]. Gauge ensembles are generated at a lattice spacing $a=0.112(1) \mathrm{fm}$ and at a strange quark mass $m_{s}=0.080$ close to its physical value $m_{s, \text { phys }}=0.081$. Four values of degenerate up and down quark masses, $m_{l}=0.015,0.025,0.035$ and 0.050 , are simulated to explore a range of the pion mass $290-540 \mathrm{MeV}$. At each $m_{l}$, we choose a lattice size, $16^{3} \times 48$ or $24^{3} \times 48$, to control finite volume effects by satisfying a condition $M_{\pi} L \gtrsim 4$. The statistics are 2,500 HMC trajectories at each simulation point $\left(m_{l}, m_{s}\right)$.

We calculate the relevant two- and three-point functions of pion and kaon by using the allto-all quark propagator. The form factors are precisely estimated from ratios of the correlation functions. We employ the twisted boundary condition for the valence quarks to simulate nearzero momentum transfers $|t| \lesssim(300 \mathrm{MeV})^{2}$. The $m_{s}$ dependence of the form factors is studied by repeating our calculation at a different $m_{s}(=0.060)$ with the reweighting technique. We refer to Refs $[3,4]$ for more details on our simulation parameters and method.

\section{EM form factors}

Chiral symmetry constrains the chiral behavior of the EM form factors. In the chiral expansion of these form factors at the next-to-leading order (NLO) in terms of the expansion parameters $\xi_{\{\pi, K\}}=M_{\{\pi, K\}}^{2} /\left(4 \pi F_{\pi}\right)^{2}$ and $t$, we have only single free parameter $L_{9}^{r}$, which is one of the 

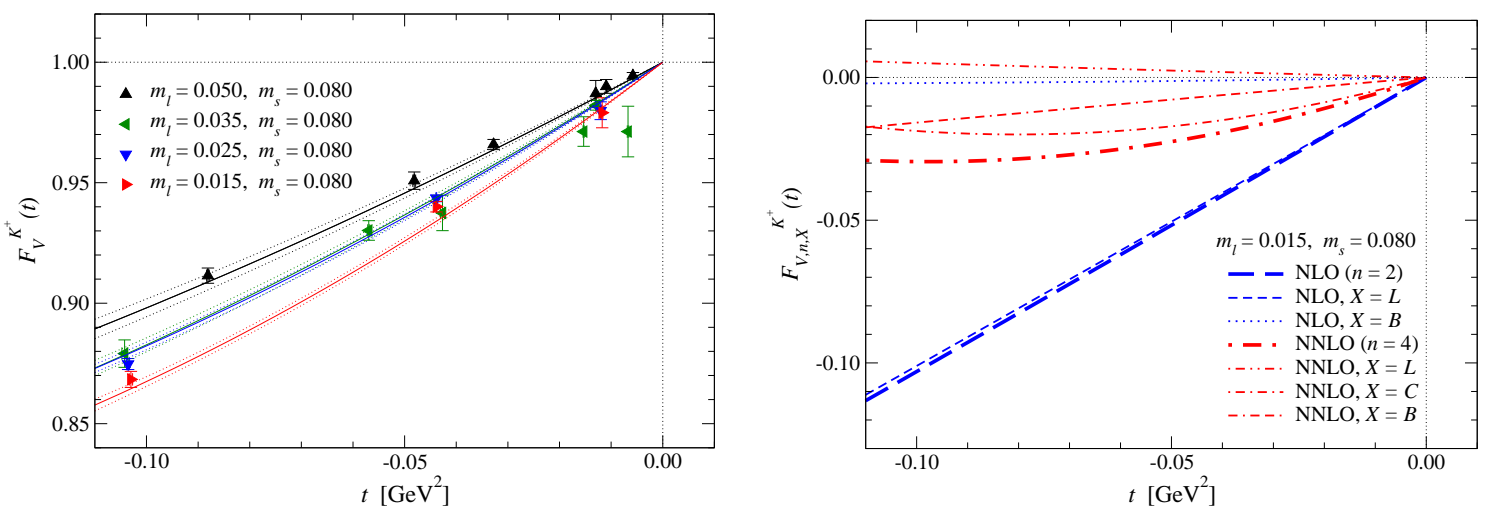

Figure 1: Left panel: NNLO ChPT fit to $K^{+}$EM form factor. Data at different $M_{\pi}$ 's are plotted in different symbols as a function of $t$. Right panel: NLO and NNLO contributions (thick lines) and their decomposition into LEC-dependent and independent parts (thin lines) at $\left(m_{l}, m_{s}\right)=(0.015,0.080)$. The blue and red lines show the NLO and NNLO contributions, respectively.

low-energy constants (LECs) in the NLO chiral Lagrangian. Many more LECs appear at NNLO: $L_{\{1, \ldots, 5\}}^{r}$ and $C_{i}^{r}$ 's in the NLO and NNLO Lagrangians. Since $L_{\{1, \ldots, 5\}}^{r}$ have been well studied in phenomenological studies of experimental data and they appear only in the possibly small NNLO corrections, we fix them to a recent phenomenological estimate in Ref. [5]. In general, $C_{i}^{r}$ 's are poorly determined in phenomenology and have to be determined on the lattice.

Let us write the NNLO chiral expansion as

$$
\begin{aligned}
F_{V}^{P}(t) & =F_{V, 0}^{P}+F_{V, 2}^{P}(t)+F_{V, 4}^{P}(t)+F_{V, 6}^{P}(t) \quad\left(P=\pi^{+}, K^{+}, K^{0}\right), \\
F_{V, 2}^{P}(t) & =F_{V, 2, L}^{P}(t)+F_{V, 2, B}^{P}(t), \quad F_{V, 4}^{P}(t)=F_{V, 4, L}^{P}(t)+F_{V, 4, C}^{P}(t)+F_{V, 4, B}^{P}(t) .
\end{aligned}
$$

The terms $F_{V, 0}^{P}, F_{V, 2}^{P}$ and $F_{V, 4}^{P}$ are the leading-order, NLO and NNLO contributions, respectively. We add an even higher order correction $F_{V, 6}^{P}$, when necessary. The additional subscripts " $L$ ", " $C$ " and " $B$ " represent $L_{i}^{r}$-dependent, $C_{i}^{r}$-dependent and LEC-independent parts, respectively. The $C_{i}^{r}$ dependent NNLO analytic terms $F_{V, 4, C}^{P}$ are given as [6]

$$
\begin{aligned}
& F_{\pi}^{4} F_{V, 4, C}^{\pi^{+}}(t)=-4 c_{\pi^{+}, \pi t}^{r} M_{\pi}^{2} t-8 c_{\pi^{+}, K t}^{r} M_{K}^{2} t-4 c_{t^{2}}^{r} t^{2}, \\
& F_{\pi}^{4} F_{V, 4, C}^{K^{+}}(t)=-4 c_{K^{+}, \pi t}^{r} M_{\pi}^{2} t-4 c_{K^{+}, K t}^{r} M_{K}^{2} t-4 c_{t^{2}}^{r} t^{2}, \\
& F_{\pi}^{4} F_{V, 4, C}^{K^{0}}(t)=-\frac{8}{3} c_{K^{0}}^{r}\left(M_{K}^{2}-M_{\pi}^{2}\right) t,
\end{aligned}
$$

where the coefficients are linear combinations of $C_{i}^{r}$ 's. These are not independent: $c_{K^{+}, \pi t}^{r}=c_{\pi^{+}, K t}^{r}+$ $c_{K^{0}}^{r} / 3$ and $c_{K^{+}, K t}^{r}=c_{\pi^{+}, \pi t}^{r}+c_{\pi^{+}, K t}^{r}-c_{K^{0}}^{r} / 3$. We therefore treat the following four as fitting parameters

$$
\begin{aligned}
& c_{\pi^{+}, \pi t}^{r}=4 C_{12}^{r}+4 C_{13}^{r}+2 C_{63}^{r}+C_{64}^{r}+C_{65}^{r}+2 C_{90}^{r}, \\
& c_{\pi^{+}, K t}^{r}=4 C_{13}^{r}+C_{64}^{r}, \quad c_{t^{2}}^{r}=C_{88}^{r}-C_{90}^{r}, \quad c_{K^{0}}^{r}=2 C_{63}^{r}-C_{65}^{r} .
\end{aligned}
$$

An example of the NNLO ChPT fit for the charged kaon EM form factor $F_{V}^{K^{+}}$is plotted in the left panel of Fig. 1. We obtain $\chi^{2} /$ d.o.f $=1.8$ and

$$
L_{9}^{r}=4.6(1.1)_{\text {stat }}\left({ }_{-0.5}^{+0.1}\right)_{L_{i}^{r}}(0.4)_{a=0} \times 10^{-3}, \quad c_{t^{2}}^{r}=-6.4(1.1)_{\text {stat }}(0.1)_{L_{i}^{r}}(0.5)_{a=0} \times 10^{-5},
$$


where the renormalization scale is set to $\mu=M_{\rho}$. The second error is the uncertainty due to the choice of the input $L_{\{1, \ldots, 5\}}^{r}$, whereas the third is the discretization error estimated by power counting $O\left(\left(a \Lambda_{\mathrm{QCD}}\right)^{2}\right) \sim 8 \%$ with $\Lambda_{\mathrm{QCD}}=500 \mathrm{MeV}$. These results are reasonably consistent with the recent phenomenological estimate in Ref. [5]. While other fit parameters are poorly known in phenomenology, our results are roughly consistent with a naive power counting $C_{i}^{r}=O\left((4 \pi)^{-4}\right)$ [4].

An interesting observation in the right panel of Fig. 1 is that the non-trivial chiral correction $F_{V, 2}^{K^{+}}+F_{V, 4}^{K^{+}}$is largely dominated by the NLO analytic term $F_{V, 2, L}^{K^{+}}$. Note that this term is not unexpectedly large, because our result for $L_{9}^{r}$ is consistent with the phenomenological estimate as well as a power counting $L_{i}^{r}=O\left((4 \pi)^{-2}\right)$. Since this term is independent of the valence quark masses $\left(F_{V, 2, L}^{\pi^{+}}=F_{V, 2, L}^{K^{+}}=2 L_{9}^{r} t / F_{\pi}^{2}\right)$, the NNLO chiral expansion of the charged meson EM form factors $F_{V}^{\left\{\pi^{+}, K^{+}\right\}}$shows reasonable convergence. This is however not the case for the neutral kaon, because $F_{V, 2, L}^{K^{0}}$ vanishes to satisfy the constraint $F_{V}^{K^{0}}(t)=0$ at $m_{l}=m_{s}$.

We obtain the following results for the charge radii from the NNLO ChPT fit

$$
\begin{aligned}
& \left\langle r^{2}\right\rangle_{V}^{\pi^{+}}=0.458(15)_{\text {stat }}\left(\begin{array}{c}
+9 \\
-1
\end{array}\right)_{L_{i}^{r}}(37)_{a \neq 0} \mathrm{fm}^{2}, \quad\left\langle r^{2}\right\rangle_{V}^{K^{+}}=0.380(12)_{\text {stat }}\left({ }_{-1}^{+7}\right)_{L_{i}^{r}}(31)_{a \neq 0} \mathrm{fm}^{2}, \\
& \left\langle r^{2}\right\rangle_{V}^{K^{0}}=-0.055(10)_{\text {stat }}(1)_{L_{i}^{r}}(4)_{a \neq 0} \mathrm{fm}^{2} .
\end{aligned}
$$

These are in reasonable agreement with the experimental values $\left\langle r^{2}\right\rangle_{V}^{\pi^{+}}=0.452(11) \mathrm{fm}^{2},\left\langle r^{2}\right\rangle_{V}^{K^{+}}=$ $0.314(35) \mathrm{fm}^{2}$ and $\left\langle r^{2}\right\rangle_{V}^{K^{0}}=-0.077(10) \mathrm{fm}^{2}[7]$.

\section{Kaon semileptonic form factors}

In Fig. 2, we demonstrate a conventional determination of the normalization $f_{+}(0)$. At simulated $M_{\pi}$ 's, we fix $f_{+}(0)$ assuming the following $t$ dependence of $f_{+}(t)$ and $f_{0}(t)$

$$
\begin{aligned}
f_{+}(t) & =f_{+}(0)\left\{\frac{1}{1-t / M_{K^{*}}^{2}}+a_{+} t\right\}, \\
f_{0}(t) & =f_{+}(0)\left(1+a_{0} t+b_{0} t^{2}\right),
\end{aligned}
$$

based on the vector meson dominance hypothesis. The results are well described by the NNLO ChPT formula with $\chi^{2} /$ d.o.f. $\sim 0.2$. The extrapolated value $f_{+}(0)=0.644(4)_{\text {stat }}$ is consistent with the average of recent results $[8,9,10]$ by the Flavor Lattice Averaging Group (FLAG) [11, 12]. We note that these recent studies are pursuing more precise

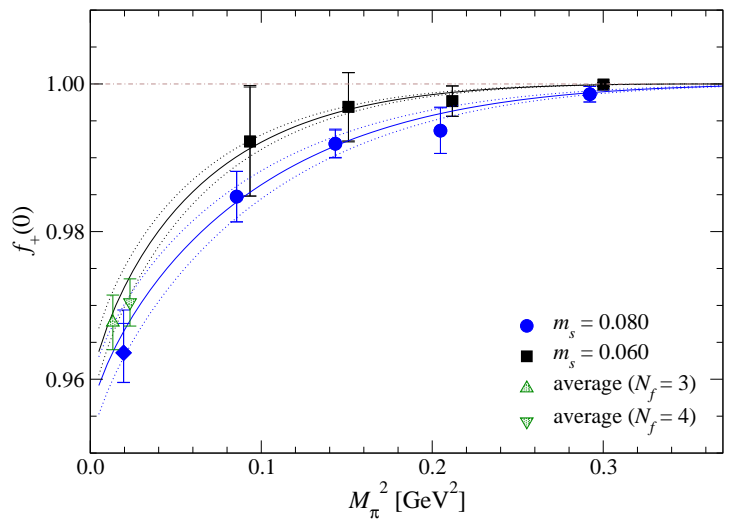

Figure 2: Extrapolation of $f_{+}(0)$ as a function of $M_{\pi}^{2}$ and $M_{K}^{2}$ based on NNLO ChPT. Circles and squares show our data at $m_{s}=0.080$ and 0.060 , respectively. The diamond represents the value extrapolated to the physical point $\left(m_{l, \text { phys }}, m_{s, \text { phys }}\right)$. We also plot the preliminary FLAG averages for $N_{f}=3$ and 4 by triangles. determination by simulations near or directly at the reference point $t=0$ and the physical point $\left(m_{l, \text { phys }}, m_{s, \text { phys }}\right)$. Note also that a study of the form factor shape based on a phenomenological parametrization is also reported at this conference [13]. 

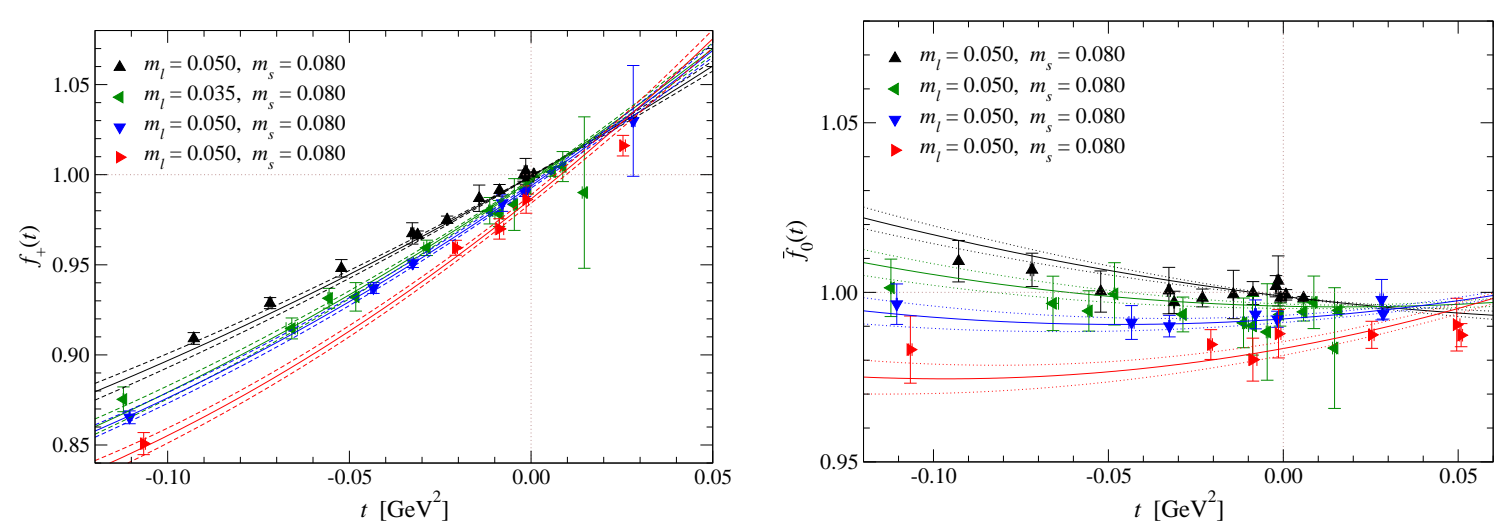

Figure 3: NNLO ChPT fit to $f_{+}(t)$ (left panel) and $\tilde{f}_{0}(t)$ (right panel) as a function of $t$. Different symbols show data at different $M_{\pi}$ 's.

We employ a different strategy exploiting exact chiral symmetry: we fit the lattice data of $f_{+}$ and $f_{0}$ to their NNLO ChPT formula as a function of $M_{\{\pi, K\}}^{2}$ and $t$. Similar to the EM form factors, the chiral expansion of the vector form factor $f_{+}[14,15]$ has only $L_{9}^{r}$ at NLO and other $L_{i}^{r}$ 's at NNLO. We fix $L_{9}^{r}$ to the value obtained in our analysis of the EM form factors, whereas others are set to the phenomenological estimate [5]. The analysis of the EM form factors provides helpful information also for the NNLO LECs. The coefficient $c_{t^{2}}^{r}$ in Eqs. (3.3)-(3.4) also appears in the NNLO analytic term of $f_{+}$as

$$
F_{\pi}^{4} f_{+, 4, C}=c_{+, \pi K}^{r}\left(M_{K}^{2}-M_{\pi}^{2}\right)+c_{+, \pi t}^{r} M_{\pi}^{2} t+c_{+, K t}^{r} M_{K}^{2} t-4 c_{t^{2}}^{r} t^{2} .
$$

Other two coefficients can be written in terms of those for the EM form factors

$$
\begin{aligned}
& c_{+, \pi t}^{r}=-4\left(2 C_{12}^{r}+4 C_{13}^{r}+C_{64}^{r}+C_{65}^{r}+C_{90}^{r}\right)=-2\left(c_{\pi^{+}, \pi t}^{r}+c_{\pi^{+}, K t}^{r}-c_{K^{0}}^{r}\right), \\
& c_{+, K t}^{r}=-4\left(2 C_{12}^{r}+8 C_{13}^{r}+2 C_{63}^{r}+2 C_{64}^{r}+C_{90}^{r}\right)=-2\left(c_{\pi^{+}, \pi t}^{r}+3 c_{\pi^{+}, K t}^{r}+c_{K^{0}}^{r}\right) .
\end{aligned}
$$

The remaining one $c_{+, \pi K}^{r}=-8\left(C_{12}^{r}+C_{34}^{r}\right)$ describes SU(3) breaking effects at $t=0$, and hence is absent in the EM form factors. Therefore we have only one free parameter $c_{+, \pi K}^{r}$ in the chiral extrapolation of $f_{+}$.

The scalar form factor $f_{0}$ has many additional NNLO LECs. In order to carry out a chiral extrapolation with less free parameters, we consider the following quantity proposed in Ref. [15]

$$
\tilde{f}_{0}(t)=f_{0}(t)+\frac{t}{M_{K}^{2}-M_{\pi}^{2}}\left(1-\frac{F_{K}}{F_{\pi}}\right) .
$$

The Dashen-Weinstein relation [16] suggests a large cancellation between the NNLO analytic terms of $f_{0}$ and $F_{K} / F_{\pi}$. In fact, the NNLO analytic term of $\tilde{f}_{0}$ is given in a rather simple form

$$
F_{\pi}^{4} \tilde{f}_{0,4, C}(t)=c_{+, \pi K}^{r}\left(M_{K}^{2}-M_{\pi}^{2}\right)+\left(8 C_{12}^{r}-c_{+, \pi K}^{r}\right)\left(M_{K}^{2}+M_{\pi}^{2}\right) t-8 C_{12} t^{2},
$$

and a simultaneous fit to $f_{+}$and $\tilde{f}_{0}$ has only two fitting parameters $c_{+, \pi K}^{r}$ and $C_{12}^{r}$.

Figure 3 shows the NNLO ChPT fit to $f_{+}(t)$ and $\tilde{f}_{0}(t)$ as a function of $t$. Our data are well described with $\chi^{2} /$ d.o.f. $\sim 0.7$. Similar to the EM form factors, the $t$ dependence of $f_{+}$is roughly 
approximated by the NLO analytic term $f_{+, 2, L}=F_{V, 2, L}^{K^{+}}$. On the other hand, $\tilde{f}_{0}$ has no NLO analytic term due to the cancellation between $f_{0}$ and $F_{K} / F_{\pi}$, and shows a rather mild $t$ dependence.

From the simultaneous fit to $f_{+}$and $\tilde{f}_{0}$, we obtain

$$
f_{+}(0)=0.9636(36)_{\text {stat }}\left({ }_{-45}^{+41}\right)_{\text {chiral }}(29)_{a \neq 0}=0.9636\left({ }_{-65}^{+62}\right)
$$

at the physical point. The first error is statistical. The second is the systematic uncertainty of the chiral extrapolation, which is estimated by repeating the fit including higher order corrections or using different values for the input $L_{\{1, \ldots, 8\}}^{r}$. The third one is the discretization error estimated by power counting. The total uncertainty is at the level of $\leq 1 \%$.

An advantage of our analysis method is that we can study both the normalization and shape of the form factors by a unique fit based on NNLO ChPT. In Fig. 4, we plot the slope $\lambda_{+}^{\prime}$ in the quadratic parametrization

$$
f_{+}(t)=f_{+}(0)\left\{1+\frac{\lambda_{+}^{\prime}}{M_{\pi^{ \pm}}^{2}} t+O\left(t^{2}\right)\right\} .
$$

Namely,

$$
\lambda_{+}^{\prime}=\left.\frac{M_{\pi^{ \pm}, \mathrm{phys}}^{2}}{f_{+}(0)} \frac{d f_{+}(t)}{d t}\right|_{t=0} .
$$

The NNLO contribution turns out to be significant even near the physical point. It is therefore important to study the form factor shape by taking account of their non-analytic chiral behavior at NNLO.

From the NNLO ChPT fit, we obtain $\lambda_{+}^{\prime}=3.08(14)_{\text {stat }}(31)_{\text {sys }} \times 10^{-2}$ and $\lambda_{0}^{\prime}=1.98(15)_{\text {stat }}(44)_{\text {sys }} \times$ $10^{-2}$, where we add the uncertainty of the chiral extrapolation and the discretization error in quadrature. This is consistent with recent experimental measurements, $\lambda_{+}^{\prime}=2.58(7) \times 10^{-2}$ and $\lambda_{0}^{\prime}=1.37(9) \times 10^{-2}[17]$ within $2 \sigma$. The largest uncertainty comes from the discretization for $\lambda_{+}^{\prime}$ and the chiral extrapolation for $\lambda_{0}^{\prime}$.

\section{Summary}

In this article, we have presented our lattice calculation of the light meson EM and semileptonic form factors. These form factors are precisely calculated by using the all-to-all quark propagator. Their chiral behavior is directory compared with continuum ChPT by exploiting exact chiral symmetry preserved with the overlap quark action.

We observe that the lattice data of the charged meson EM form factors are reasonably well described by their NNLO ChPT formula, and estimate the relevant NLO and NNLO LECs. These results are used in the chiral extrapolation for the kaon semileptonic form factors, and their normalization $f_{+}(0)$ is determined with sub- $\%$ accuracy. We confirm reasonable consistency of the form factor shape, namely the charge radii and slopes of the semileptonic form factors, with experiment. 
One of the largest uncertainty is the discretization error at a finite lattice spacing. It is important to extend this study to finer lattices and heavy flavor physics. Simulations in these directions are underway [18] using a computationally cheaper fermion action with good chiral symmetry [19].

We thank Johan Bijnens for making his code to calculate pion and kaon form factors in NNLO ChPT available to us. Numerical simulations are performed on Hitachi SR16000 and IBM System Blue Gene Solution at KEK under a support of its Large Scale Simulation Program (No. 15/16-09), and on SR16000 at YITP in Kyoto University. This work is supported in part by the Grant-in-Aid of the MEXT (No. 25287046, 26247043, 26400259 and 15K05065) and by MEXT SPIRE and JICFuS.

\section{References}

[1] H. Fukaya et al. (JLQCD Collaboration), Phys. Rev. D 74, 094505 (2006) [arXiv:hep-lat/0607020].

[2] S. Aoki, H. Fukaya, S. Hashimoto and T. Onogi, Phys. Rev. D 76, 054508 (2007) [arXiv:0707.0396 [hep-lat]].

[3] T. Kaneko et al. (JLQCD Collaboration), PoS Lattice 2012111 (2012) [arXiv:1211.6180 [hep-lat]].

[4] S. Aoki et al. (JLQCD Collaboration), arXiv:1510.06470 [hep-lat].

[5] J. Bijnens and G. Ecker, Ann. Rev. Nucl. Part. Sci. 64, 149 (2014) [arXiv:1405.6488 [hep-ph]].

[6] J. Bijnens and P. Talavera, JHEP 0203, 046 (2002) [arXiv:hep-ph/0203049].

[7] K.A. Olive et al. (Particle Data Group), Chin. Phys. C, 38, 090001 (2014).

[8] A. Bazavov et al. (Fermilab Lattice and MILC Collaborations), Phys. Rev. D 87, 073012 (2013) [arXiv:1212.4993 [hep-lat]].

[9] A. Bazavov et al. (Fermilab Lattice and MILC Collaborations), Phys. Rev. Lett. 112, 112001 (2014) [arXiv:1312.1228 [hep-ph]].

[10] P.A. Boyle et al. (RBC/UKQCD Collaboration), arXiv:1504.01692 [hep-lat].

[11] S. Aoki et al. (Flavor Lattice Averaging Group), in preparation.

[12] A. Jüttner, in these proceedings.

[13] L. Riggio et al. (ETM Collaboration), in these proceedings.

[14] P. Post and K. Schilcher, Eur. Phys. J. C 25, 427 (2002) [arXiv:hep-ph/0112352].

[15] J. Bijnens and P.Talavera, Nucl. Phys. B 669, 341 (2003) [arXiv:hep-ph/0303103].

[16] R.F. Dashen, L. Ling-Fong, H. Pagels and M. Weinstein, Phys. Rev. D 6, 834 (1972).

[17] M. Moulson, arXiv:1411.5252 [hep-ex].

[18] J. Noaki et al. (JLQCD Collaboration), PoS LATTICE2014, 069 (2015).

[19] T. Kaneko et al. (JLQCD Collaboration), PoS LATTICE2013, 125 (2014) [arXiv:1311.6941 [hep-lat]]. 